$10.2478 / \mathrm{gb}-2020-0002$

sciendo

\title{
Allmählich clowngesichtig. Zu einer literarischen \\ Maske des modernen Autors \\ am Beispiel Paul Celans
}

\section{Laura CHEIE}

Doz. Dr., West-Universität Temeswar/Timișoara);

E-Mail: laura.cheie@gmail.com

\begin{abstract}
Most of the times the tragic mask of the madman has fascinated artistic and literary modernity, inspiring a series of painters and writers through its complex expresivity and semantics. Balancing between lie and truth, covering and revelating, through anarchy and virtuosity, the mask of the tragic clown reflects loneliness, the anxieties and demones of the modern individual in a world of violence and alienation. In Celan's late poetry, the mask of the tragic clown describes another kafkian metamorphosis which the present paper analyses in a cultural, biographic and intertextual context.
\end{abstract}

Key words: mask, clown, poetic identity, metamorphosis

In der Einleitung seines Essaybandes Masken und Stimmen. Figuren der modernen Lyrik aus dem Jahr 1996 bemerkt der Dichter und Literaturkritiker Harald Hartung:

Der Dichter, der sich maskiert, versucht unter der Maske seine authentische Stimme zu finden. Der Möglichkeiten, der Varianten sind viele: sie reichen von den Personae Ezra Pounds und dem Personenpluralismus der Heteronyme Fernando Pessoas bis in die linguistischen Exerzitien Helmut Heißenbüttels, Oskar Pastiors oder Inger Christensens; von der Ausdrucksartistik Gottfried Benns bis zum reduktionistischen grido Giuseppe Ungarettis; von der nüchternen Wahrheit Philip Larkins bis zu den kühnen Metaphern, unter denen 
Tomas Tranströmer den Kampf um den verlorenen Namen führt. Und der Name, unter dem Paul Antschel im anagrammatischen Silbentausch zu Paul Celan wurde, bot er nicht so viel Schutz, daß in seinem Schatten Atem und ,Atemwende möglich wurden?'

Für Hartung gehört somit die Maske, die bereits im Pseudonym sichtbar werden soll, zur dichterischen Identität des modernen Poeten, weil sie ihm den notwendigen ,Schutz“ verleihe, seine ,authentische Stimme“ zu suchen und zu finden. Dass Masken im konkreten und metaphorischen Sinn eine Schutzfunktion erfüllen können oder dass ihnen eine solche Funktion zugedacht wird, lässt sich unschwer belegen. Das ist der Fall mancher konkreter Maskierungen, wie zum Beispiel in der Medizin, und metaphorisch interpretierter körperlicher oder sprachlicher Haltungen, die durch gesellschaftliche, kulturelle und sehr wohl auch literarische Normen, Techniken und Kontexte entstehen, wie eben Formen sozialer Höflichkeit oder die Wahl eines Pseudonyms. Dass Masken aber, die vielmehr als Symbol für das Künstliche fungieren, Authentisches hervorbringen sollen, klingt paradox. Allerdings entspricht die Neigung zum Paradoxen der Gestalt, Wirkung und Auffassung von Masken, wie Richard Weihe in seiner nuancierten Untersuchung zu deren Geschichte und Semantik feststellt. ${ }^{2}$

Die Maske im eigentlichen und im übertragenen Sinn ist, wie Weihe bemerkt, auf eine komplexe Weise ambivalent und ihre Ambivalenz beruht oft auf Paradoxem. Sie unterliegt konkret, als ,Zwei-Seiten-Form“33, aber auch in ihrer metaphorischen Verwendung, beispielsweise des zur Maske gewordenen Gesichts, einer grundsätzlichen Dialektik von Zeigen und

${ }^{1}$ Hartung, Harald: Masken und Stimmen. Figuren der modernen Lyrik. München/Wien: Hanser 1996, S. 27.

2 Weihe, Richard: Die Paradoxie der Maske. Geschichte einer Form. München: Fink 2004.

3 Den Begriff übernimmt Weihe von Niklas Luhmann. Vgl. Weihe 2004, S. 43. 
Verhüllen. „Sie zeigt, indem sie verbirgt ${ }^{“ 4}$, sie stellt dar und/ oder kommuniziert etwas, indem sie aber das wahre Gesicht, die eigentliche Identität verdeckt. Diese Tarnungsfunktion brachte das „künstliche“ Gesicht spätestens seit dem Christentum in Verbindung mit den Ideen der Täuschung und der Lüge, was die Maske zum negativen Symbol der Verstellung, zum „Inbegriff des Falschen“, ja des Diabolischen ${ }^{5}$ werden ließ. Doch so einfach lassen sich Masken nicht in die Logik des Entwederoder zwingen, denn, wie Weihe bemerkt, ist die Maske „sowohl das Innen als auch das Außen. Indem sie zugleich trennt und verbindet, können wir die Maske als Gleichzeitigkeit des Entweder-oder und des Sowohl-als-auch verstehen". ${ }^{6}$ Masken trennen und verbinden dabei nicht nur eine Innen- und eine Außenseite, sondern überlagern mindestens zwei Identitäten und können neben Ambivalenz auch Verwandlung darstellen. Sie sind selbst höchst wandelbar und veranschaulichen ebenfalls, so Weihe, „das Angebot, sich zu verändern. Sie versprechen: andere Gesichter, andere Existenzen, andere Identitäten. Masken ermöglichen es, das Ich als Rollenspiel und die Persönlichkeit als eine vielgesichtige, facettierte zu begreifen. ${ }^{67}$ Es ist daher kaum befremdlich, dass sie moderne Künstler und Autoren faszinierte.

Unter den beliebtesten Masken der modernen Kunst und Literatur von der Jahrhundertwende bis ins einundzwanzigste Jahrhundert befindet sich jene des Narren, genauer des Zirkusnarren in seinen verschiedenen Variationen als Pierrot, Harlekin oder Clown. Als Symbol des frei agierenden, absoluten Künstlers wird er zur quasi obsessiven Identifikationsfigur für Picasso, erscheint aber auch in Gemälden von Rouault, Cézanne, Renoir, Gris, Miró, Dalí, Beckmann, Macke, Klee, Picabia, Chagall, Toulouse-Lautrec etc. In der modernen deutschsprachigen

\footnotetext{
${ }^{4}$ Weihe 2004, S. 14.

5 Weihe 2004, S. 32, 53.

6 Weihe 2004, S. 46-47.

7 Weihe 2004, S. 16.
} 
Literatur entwickelt sich der Clown zum „modernen alter ego des Schriftstellers" ${ }^{\text {"8 }}$. So greifen beispielsweise Karl Emil Franzos in seinem Roman Der Pojaz und der junge Thomas Mann in seiner Novelle Der Bajazzo zur Maske des Zirkusclowns. Hugo von Hofmannsthal schreibt für die Oper Ariadne auf Naxos ein Liedchen des Harlekin und Heinrich Böll publiziert 1963 seinen berühmten Roman Ansichten eines Clowns, in welchem er in der Maske des Zirkusnarren die Verlogenheit der Mitläufer des Nationalsozialismus in der Nachkriegszeit kritisiert.

Doch was genau für eine Narrenmaske ist der Clown? Der Duden definiert den Clown als „Spaßmacher im Zirkus, Varietéc" allerdings sind Clowns nicht lediglich Kunstfiguren der Zirkuswelt, denn, wie Richard Weihe bemerkt: „Der Clown hat im Lauf seiner Kulturgeschichte verschiedene physische und imaginäre Räume kolonisiert: Theater, Zirkus, Music Hall, Varieté, Film, Fernsehen, Literatur, Comic, Oper, Anthropologie. “" Das englische Wort „clown“ bezeichnete ursprünglich, also in der zweiten Hälfte des 16. Jahrhunderts, eine Gegenfigur zum Gentleman, also einen Bauern oder Landmann (,rustic") als einen Tölpel und Grobian, wodurch er auch zum Äquivalenten des Narren wurde. ${ }^{11}$ Den Clown verstand man dabei sowohl als „fool“, also als Narr, wie auch als ,jester“, also als Spaßvogel,

8 Velten, Hans Rudolf: Spaßmacher. In: Wirth, Uwe (Hg.): Komik. Ein interdisziplinäres Handbuch. Stuttgart: Metzler 2017, S. 42-46, hier S. 45. Zum modernen Künstler und Schriftsteller als Clown vgl. auch Jean Starobinski: Porträt des Künstlers als Gaukler. Drei Essays. Aus dem Französischen von Markus Jacob. Frankfurt am Main: S. Fischer 1985.

9 Duden: Clown. https://www.duden.de/rechtschreibung/Clown [20.10. 2019]

${ }^{10}$ Weihe, Richard: Das (Un-)Behagen am Clown. Einleitung. In: Weihe, Richard (Hg.): Über den Clown. Künstlerische und theoretische Perspektiven. Bielefeld: transcript Verlag 2016a, S. 7-21, hier S. 8-9.

${ }^{11}$ Vgl. Weihe 2016a, S. 11. Vgl. auch Barloewen, Constantin: Clown. In: Hügel, Hans-Otto (Hg.): Handbuch Populäre Kultur. Begriffe, 
doch seine Hauptbedeutung war die einer einfacheren, ja triebhaften Gestalt, die noch an den derben Witzbold mittelalterlicher Fastnachtsumzüge erinnerte. In Shakespeares Dramen ( $A s$ You Like it, The Merchant of Venice, A Midsummer Night's Dream, Much Ado About Nothing) erscheinen Clowns, die, mit Dimiter Daphinoff gesprochen, an das eher triebhafte Leben gebunden sind. ${ }^{12}$ Weitere Ahnen des Clowns kommen aus der Commedia dell'arte. So ist bereits an der Maske des Weißclowns seine Verwandtschaft mit dem weiß geschminkten romantischen Pierrot zu erkennen. Das bunte Kostüm des dummen August erinnert an Arlecchino, während ihn sein plumpes Verhalten als Nachfolger des Brighella ausweist. ${ }^{13}$

Kennzeichen des modernen Clowns ist vor allem eine vielfältige Widersprüchlichkeit. Laut Weihe ist er zunächst ein Grenzgänger, der entlang der, aber auch über konkrete und politische oder gesellschaftliche Grenzen hinweg spielt. Er vereint in sich sowohl das Kind als auch den Erwachsenen, die extreme Ungeschicklichkeit wie auch eine virtuose Akrobatik, das Männliche und das Weibliche. Darüber hinaus vereint der Clown Lachen und Weinen, eine virtuose Sprachbeherrschung wie auch Sprachlosigkeit, Stottern oder ein rudimentäres Sprachvermögen und verwischt schließlich den Gegensatz von Gut und Böse, indem er als gutmütiger Narr, aber auch als verstörender Horrorclown auftreten kann. ${ }^{14}$ Für Constantin von

Theorien und Diskussionen. Stuttgart/Weimar: J. B. Metzler 2003, S. 134-138, hier S. 134.

${ }^{12}$ Daphinoff, Dimiter: Shakespeares Narren. In: Huber, H. (Hg.): Der Narr: Beiträge zu einem interdisziplinären Gespräch. Freiburg, Schweiz: Univ.-Verl. 1991 (= Studia ethnographica Friburgensia. Bd. XVII), S. 57-69, hier S. 63.

${ }^{13}$ Vgl. Barloewen 2003, S. 136.

${ }^{14}$ Weihe, Richard: Die Paradoxie des Clowns - sieben Spielformen. In: Weihe, Richard (Hg.): Über den Clown. Künstlerische und theoretische Perspektiven. Bielefeld: transcript Verlag 2016b, S. 265-274 passim. 
Barloewen ist der Clown ein „konstruktiver Anarch" ${ }^{\text {"15, der sich }}$ zwischen Einhaltung und Auflösung der Norm bewegt und der gerade im Stolpern, ,in diesem vermeintlichen Scheitern, an Größe gewinnt ${ }^{\text {“16, }}$, weil er auch die Hoffnung verkörpert, ,,dass nach dem Absturz wieder ein Aufstieg folgt ${ }^{\text {"17 }}$. Allerdings spiegeln manche Clowns der Kunst und Literatur dieses Selbstvertrauen im Gelingen des Falls und des Aufstehens danach nicht wider. Von einer besonderen Eindringlichkeit sind diesbezüglich die Bilder des tragisch-düsteren Clowns, wie sie in der Malerei beispielsweise bei Georges Rouault (Tête de clown tragique, um 1904/1905) oder bei Francis Picabia (Le Clown Fratellini, 1937-1938), im Film zum Beispiel in Federico Fellinis La Strada (1954), in der Literatur bei Heinrich Böll im Roman Ansichten eines Clowns (1963) erscheinen. Der tragische Clown, eine somit beliebte Identifikationsfigur des modernen Künstlers und Schriftstellers, widerspiegelt die Einsamkeit, die Ängste und Dämonen, ja das Scheitern des modernen Individuums in einer Welt der Gewalt, der affektiven Entfremdung und Kälte.

In der Tradition des tragischen Clowns steht auch Celan mit seinem späten Gedicht Allmählich clowngesichtig aus dem postum erschienenen Band Lichtzwang (1970):

Allmählich clowngesichtig,

nichtsgespiegelt,

die Schminke Wahrheit blaugefrorn im Winkelmund,

Frostpollen Puder auf dem blanken Überschädel, rund um die dünne Fragelocke Schwarz,

${ }^{15}$ Barloewen, Constantin von: Der Clown als konstruktiver Anarch. Reflexionen über die Dialektik des Clowns. Constantin Barloewen im Gespräch mit Rafiu Raji und Richard Weihe. In: Weihe, Richard (Hg.): Über den Clown. Künstlerische und theoretische Perspektiven. Bielefeld: transcript Verlag 2016, S. 127-136, hier S. 129.

${ }^{16}$ Barloewen 2016, S. 130.

${ }^{17}$ Ebenda, S. 132. 
die Brauen, Brauen: wachsend, zwei Riesenfühlerkämme, zwei,

- du großgestrählte,

großgespürte Rauhnacht Immerimmer -

schon fortgeschwungen aus der Flocke Welt, nicht hin, nicht her. ${ }^{18}$

Das Gedicht entstand in Paris, am 5. November $1967^{19}$, achtzehn Tage vor seinem 47. Geburtstag. Zu Beginn jenes Jahres hatte Celan versucht, sich durch einen Stich ins Herz das Leben zu nehmen. Am 30. Januar wurde er jedoch in einer Pariser chirurgischen Klinik durch eine Notoperation an der schwer verletzten linken Lunge gerettet. ${ }^{20}$ Der misslungene Selbstmordversuch zeigte aber einen durch die langjährige, von Claire Goll gegen ihn initiierte Plagiatskampagne erheblich fragilisierten Dichter. Claire Golls Bemühungen, Celan vor Schriftstellern, Kritikern und Verlagshäusern zu diskreditieren, empfand er als Versuch eines dreifachen Auslöschungsverfahrens, das ihn als Dichter, als Jude und als rational denkenden Menschen mundtot machen sollte. ${ }^{21}$ In diesem Kontext, wie auch in dem der Schuld abweisenden oder verkennenden 1950er und 1960er Nachkriegsjahre in Deutschland, ist es nicht befremdlich, dass „Wahrheit“" für den jüdischen Dichter aus der Bukowina zu einem „Gegenbegriff: weil es der Grundbegriff ist" wird, wie er Ingeborg

${ }^{18}$ Celan, Paul: Die Gedichte. Kommentierte Gesamtausgabe in einem Band. Hrsg. v. Barbara Wiedemann, Frankfurt am Main: Suhrkamp 2003, S. 298-299. Im Folgenden KG abgekürzt.

${ }^{19} \mathrm{Vgl}$. Wiedemann in KG, S. 822.

${ }^{20}$ Vgl. Horn, Peter: Die Garne der Fischer der Irrsee. Zur Lyrik von Paul Celan. Oberhausen: Athena 2011, S. 201.

${ }^{21}$ Vgl. diesbezüglich seinen Brief vom 8. Februar 1962 an Alfred MargulSperber in: Solomon, Petre: Paul Celan. Dimensiunea românească. Bukarest: Kriterion 1987, S. 261-263. Vgl. auch erläuternd dazu: Wiedemann, Barbara: Paul Celan - Die Goll-Affäre: Dokumente zu einer ,Infamie“. Frankfurt am Main: Suhrkamp 2000. 
Bachmann in einem Brief aus dem Jahr 1957 schreibt. ${ }^{22}$ Und dass er das Gedicht als „Dokument einer Krise [...] und zwar radikal" betrachtet. ${ }^{23}$ Ende der 1960er Jahre fühlte sich der psychisch zunehmend labile Dichter missverstanden, verraten, vereinsamt und missbraucht, ein Einzelkämpfer auf verlorenem Posten. Im Februar 1967 wurde Celan bereits zum zweiten Mal in eine psychiatrische Klinik eingewiesen, die er erst im Mai wieder verließ. Als ihn sein rumänischer Freund Petre Solomon im Sommer desselben Jahres in Paris besuchte, fand er ihn

stark verändert, vorzeitig gealtert, wortkarg, mürrisch. [...] ,Man macht Experimente mit mir', sagte er mit erstickter Stimme, aufseufzend wahrscheinlich wegen der erlittenen Schocktherapie und der ihm verabreichten Beruhigungsmittel. [...] Paul war nicht die ganze Zeit deprimiert, manchmal hatte er freudige Momente - aber freilich sehr kurze, und unterbrochen von einem nervösen Lachen, das schrill und zerstört klang. ${ }^{24}$

$\mathrm{Zu}$ diesem Gemütszustand passt die Maske des tragischen Clowns. Celan scheint allerdings eine ambivalente Beziehung zu Masken gehabt zu haben. 1951 schreibt er an Ingeborg Bachmann: „Manchmal kommt einem das Gedicht vor wie eine Maske, die es nur darum gibt, weil die anderen dann und wann ein Ding brauchen, hinter dem sie ihre heiliggesprochenen Alltagsfratzen verbergen können." ${ }^{25}$ Er verstand somit die Maske als Tarnung, Verstellung, ja Lüge und maskenhafte Gedichte als heuchlerisch. Trotzdem schlüpft er immer wieder

${ }^{22}$ Bachmann, Ingeborg/ Paul Celan: Herzzeit. Der Briefwechsel, hrsg. u. kommentiert von Bertrand Badiou, Hans Höller, Andrea Stoll und Barbara Wiedemann, Frankfurt am Main: Suhrkamp 52016, S. 64.

${ }^{23}$ Vgl. Brief an Bachmann vom 21. September 1963 in: Bachmann/ Celan ${ }^{5} 2016$, S. 158-159.

${ }^{24}$ Solomon 1987, S. 167-169. Vgl. dazu die deutsche Übersetzung in: Felstiner, John: Paul Celan. Eine Biographie. Deutsch von Holger Fliessbach, München: C.H.Beck 1997, S. 311.

${ }^{25}$ Brief an Bachmann vom 30. Oktober 1951 in: Bachmann/Celan ${ }^{5} 2016$, S. 34-36, hier S. 35. 
in die (Sprach-)Maske des Narren, wie z. B. in den Gedichten Tübingen, Jänner; Eine Gauner- und Ganovenweise und Huhediblu aus dem Band Die Niemandsrose (1963), in Schief aus dem Band Fadensonnen (1968), in Holzgesichtiger aus dem Band Schneepart (1971). Das sprachliche Spiel mit der Maske des Narren zeugt somit von einer sehr wohl identifizierbaren Faszination vor dem bezüglich des ,künstlichen Gesicht(s)“.

In Allmählich clowngesichtig ist die Maske des Clowns texttragend. Dabei deutet der Einstiegsvers auf eine sich langsam vollziehende Transformation hin, die zunächst darauf anspielt, dass das Gesicht zunehmend von einer Maske verdeckt, ja ersetzt wird oder sich maskenhaft verändert. Das Wort „clowngesichtig“ ist, wie Irene Fuß1 bemerkt, ambivalent, aber nicht unbedingt, weil es auf ,zwei Gesichter (ein natürliches und ein aufgemaltes Gesicht, eine Maske) [...] oder das Zweite Gesicht zu haben“"26 verweisen könnte. Vielmehr legt es ein Schwanken zwischen Gesicht und Maske nahe. Die Maske ist in den ersten sechs Verszeilen skizzenhaft, an einzelnen Details - Schminke, Winkelmund, Frostpollen, Puder, Überschädel, dünne Fragelocke Schwarz - deutlich zu erkennen. Das Gesicht eines Pierrot oder eines Weißclowns scheint sich hier sprachlich abzuzeichnen. Pierrot und sein späterer Nachfolger, der Weißclown, spiegeln durch ihre weißgeschminkten Gesichter die Blässe des Todes. Annette Fried und Joachim Keller deuten deswegen den Weißclown als „Bote aus einem Totenreich, ein Reisender zwischen jenseitiger Zeitlosigkeit und diesseitiger Zeitlichkeit ${ }^{\text {" } 27}$, was ihm erlaube mit verschiedenen Formen der

${ }^{26}$ Fuß1, Irene: ,, Geschenke an Aufmerksame“. Hebräische Intertextualität und mystische Weltauffassung in der Lyrik Paul Celans. Tübingen: Niemeyer 2008, S. 69.

${ }^{27}$ Annette Fried und Joachim Keller deuten deswegen den Weißclown als „Bote aus einem Totenreich, ein Reisender zwischen jenseitiger Zeitlosigkeit und diesseitiger Zeitlichkeit“. Fried, Annette/ Keller, Joachim: Faszination Clown. Düsseldorf: Patmos 1996, S. 190. 
Zeitwahrnehmung zu jonglieren. ${ }^{28}$ Zugleich stiftet er eine „Beziehung zwischen vorderhand Zusammenhanglosem und zerreißt Sinnbezüge durch seine eigenwillige Auslegung logischer Gesetzmäßigkeiten. “29 Wenn aber Clowns durch ihre Maske bestimmte Gefühle, Befindlichkeiten, Gedanken spiegeln, so heißt es bei Celan in der zweiten Verszeile „,nichtsgespiegelt“. Die Maske oder das maskenhafte Gesicht scheint ausdruckslos und starr zu sein.

Doch diese Ausdruckslosigkeit trügt, denn das Maskenhafte verweist nicht nur auf eine Verwandlung, sondern gleichzeitig auf eine Degradierung, die durch die bitter-ironische Gleichsetzung von „Schminke“ und „Wahrheit“ veranschaulicht wird. Eine auf „Schminke“, also auf das Künstlich-Oberflächliche reduzierte Wahrheit zeigt, wie es um die Macht der letzteren bestellt ist. Das kraftvolle Sprachbild der ohnmächtigen Wahrheit wird zusätzlich durch jenes der Erstarrung - ,blaugefrorn / im Winckelmund“ - verstärkt. Der Dichter kombiniert hier das Bildparadigma des Maskenhaften mit einem anderen, der Kälte: die „Schminke Wahrheit" ist „blaugefrorn“"30, „Frostpollen Puder" liegen auf dem Überschädel.

In Celans Dichtung sind Bilder der Kälte rekurrent. Schneegedichte über eine innerliche und äußerliche Eiszeit sind nicht erst ab dem postum erschienenen Gedichtband Schneepart (1971), in dem sich Schnee und Eis tatsächlich zu einem dominanten Motivkomplex entwickeln ${ }^{31}$, zu verzeichnen. Sie erscheinen, beginnend mit Schwarze Flocken, bereits seit dem

${ }^{28}$ Fried/Keller 1996, S. 203.

${ }^{29}$ Ebenda, S. 200.

${ }^{30}$ In einer Variante hieß es noch deutlicher ,festgefror(e)n“. Vgl. Celan, Paul: Lichtzwang. Vorstufen - Textgenese - Endfassung. Bearbeitet von Heino Schmull unter Mitarbeit von Markus Heilmann und Christiane Wittkop, Frankfurt am Main: Suhrkamp 2001, S. 152. (Tübinger Ausgabe).

${ }^{31}$ Vgl. dazu Amthor, Wiebke: Schneepart. In: May, Markus/ Peter Goßens/ Jürgen Lehmann (Hgg.): Celan-Handbuch. Leben - Werk 
frühen Band Der Sand aus den Urnen (1948), verstärkt jedoch in der Spätlyrik. ${ }^{32}$ Der Band Lichtzwang, zu dem, wie erwähnt, das besprochene Gedicht gehört, führt den Leser bereits mit einem der ersten Gedichte - Muschelhaufen - zurück in die ausgehende Eiszeit des Altpaläolithikums ${ }^{33}$. In den 1960er und 1970er Jahren war die Eiszeit auch unter Klimaexperten ein heftig debattiertes Thema. Generell grassierte in jenen Jahrzehnten eine Angst vor einer neuen Kaltzeit ${ }^{34}$, über welche Celan selbst im „Spiegel“ gelesen hatte, wie der Briefwechsel mit Gisela Dischner bezeugt. ${ }^{35}$ Die Kälte gehörte ebenfalls zur allgemeinen Thematik der Literatur in den 1960er Jahren. Thomas Bernhard publiziert 1963 seinen Roman Frost und

- Wirkung. Stuttgart / Weimar: J. B. Metzler 22012, S. 115-123, besonders S. 119.

${ }^{32}$ Vgl.zur Semantik von Schnee, Eis und Gletscher in Celans Lyrik: Pöggeler, Otto: Spur des Worts. Zur Lyrik Paul Celans. München: Karl Alber 1986; Pöggeler, Otto: Der Stein hinterm Aug. Studien zu Celans Gedichten. München: Fink 2000; Werner, Uta: Textgräber. Paul Celans geologische Lyrik. München: Fink 1998.

${ }^{33}$ Vgl. Sieghild Bogumil-Notz: Lichtzwang. In: May, Markus/ Peter Goßens/ Jürgen Lehmann (Hgg.): Celan-Handbuch. Leben - Werk Wirkung. Stuttgart, Weimar: J. B. Metzler ${ }^{2} 2012$, S. 105-115, hier S. 108. Vgl. auch Wiedemann, Barbara: ,LESEN SIE! IMMERZU NUR LESEN“: Celan-Lektüre und Celans Lektüren. In: „Poetica“. Bd. 36, Nr. 1/2 (2004), S. 169-191, hier S. 174-175.

${ }^{34}$ Vgl. die diesbezüglichen Ausführungen in: Behringer, Wolfgang: Kulturgeschichte des Klimas: Von der Eiszeit bis zur globalen Erwärmung. München: C.H.Beck ${ }^{5} 2010$, S. 247.

${ }^{35}$ Vgl. Celan, Paul/ Gisela Dischner: Wie aus weiter Ferne zu Dir. Briefwechsel. Hrsg. v. Barbara Wiedemann, Frankfurt am Main: Suhrkamp 2012. Nicht nur die Zeitschrift Spiegel brachte Beiträge über künftige oder vergangene Eiszeiten sondern auch andere, beispielsweise Die Zeit. Vgl. Blumen aus der Eiszeit. In: Die Zeit, Nr. 47/1967: https:// www.zeit.de/1967/47/blumen-aus-der-eiszeit/komplettansicht [03.10.2019]; Eine Eiszeit pro kosmisches Jahr. In: Die Zeit, Nr. 51/1967, https://www.zeit.de/1967/51/eine-eiszeit-pro-kosmischesjahr [03.10.2019]. Vgl. zu Celan als Leser der Tagespresse: Wiedemann 2004, S. 189-190 passim. 
Guntram Vesper den Gedichtband Am Horizont die Eiszeit, Luise Kaschnitz schreibt 1964 über körperliche und seelische Kälte in der Erzählung Die Füße im Feuer, 1967 bringt Georg von der Vring seinen Gedichtband Gesang im Schnee heraus und Alfred Andersch veröffentlicht 1969 die Reisebeschreibung Hohe Breitengrade oder Nachrichten von der Grenze. Die Kälte ist, wie Silvio Vietta hervorhebt, ein zentrales Bildfeld der ästhetischen Moderne überhaupt, eine Epochenmetapher, mit der Schriftsteller wie Hölderlin, Büchner, Kafka oder Bernhard auf eine Epoche vielfacher Verlusterfahrungen - des äußeren wie inneren Wärme- und Lebensverlustes, des drohenden Sprachverlustes ${ }^{36}$, der metaphysischen „Einsamkeit und Obdachlosigkeit" durch den Transzendenzverlust ${ }^{37}$ - reagieren. Es ist die Metapher für den kritischen, ,radikal desillusionierten Blick ${ }^{638}$, zu dem der unterkühlte jedoch intensive Ausdruck sprachlicher Lakonik ${ }^{39}$ passt, der in Celans Spätlyrik dominiert und auch die gleichzeitig abrupte wie verdichtete Sprache des Gedichts Allmählich clowngesichtig kennzeichnet.

In die starre und kalte Clownsmaske Celans kommt jedoch Bewegung. Mit dem Wachsen der Brauen und ihrer Transformation in „zwei Riesenfühlerkämme“ entpuppt sich die Maske als Larve, als Medium einer Metamorphose, die nicht zufällig an Kafkas ungeheures Ungeziefer erinnert. Die von Irene Fuß1 ebenfalls bemerkte Annäherung an Kafkas ,überdimensionalen

${ }^{36}$ Vietta, Silvio: Die Modernekritik der ästhetischen Moderne. In: Vietta, Silvio/ Dirk Kemper (Hgg.): Ästhetische Moderne in Europa. Grundzüge und Problemzusammenhänge seit der Romantik. München: Fink 1998, S. 531-549 passim.

${ }^{37}$ Vietta, Silvio: Die literarische Moderne. Eine problemgeschichtliche Darstellung der deutschsprachigen Literatur von Hölderlin bis Thomas Bernhard. Stuttgart: J. B. Metzler 1992, S. 184.

${ }^{38}$ Vietta 1998, S. 540.

${ }^{39}$ Vgl. dazu meine eingehenderen Untersuchungen zur lakonischen Lyrik: Cheie, Laura: Harte Lyrik. Zur Psychologie und Rhetorik lakonischer Dichtung in Texten von Günter Eich, Erich Fried und Reiner Kunze. Innsbruck: Studienverlag 2010. 
Käfer"40 kann sehr wohl auf Celans lebenslange Auseinandersetzung mitdem Werk Kafkas zurückgehen. ${ }^{41}$ InKafkas Verwandlung wird bekanntlich der Protagonist Gregor Samsa buchstäblich über Nacht zu einem nicht weiter identifizierten Insekt. Doch diese Metamorphose betrifft nicht nur ein Individuum, sondern bewirkt zugleich eine Inversion der Verhältnisse in Samsas Familie, wie Sandra Poppe bemerkt: Vor Gregors Verwandlung ist es ,seine Familie, die sich parasitär von ihm ernährt. Sein eigenes ,Parasitentum ' beginnt erst mit der Verwand-

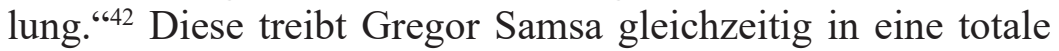
und schließlich fatale Isolation. Bildlicher Ausdruck dafür ist Samsas Panzer, der zugleich für Unbeweglichkeit und Erstarrung steht und im Konkreten die seelische Befindlichkeit des Protagonisten darstellt. ${ }^{43}$

Auch Celan wurde im Juni 1967, nach der Entlassung aus der psychiatrischen Klinik, in die Isolation getrieben. Auf Wunsch seiner Frau Gisèle Celan-Lestrange, die er in einem Wahnzustand attackiert hatte, sollte Celan, vor allem zum Schutz

${ }^{40}$ Fuß1 2008, S. 70-71.

${ }^{41}$ Vgl. zur diesbezüglichen Forschungsliteratur: Sparr, Thomas: Celan und Kafka. In: Celan-Jahrbuch 2 (1988), S. 140-154; Günzel, Elke: Das wandernde Zitat. Paul Celan im jüdischen Kontext. Würzburg: Königshausen \& Neumann 1995; Liska, Vivian: Ein Meridian wider die Zeit. Von Celan zu Kafka. In: Engel, Manfred/ Dieter Lamping (Hgg.): Franz Kafka und die Weltliteratur. Göttingen: Vandenhoeck \& Ruprecht 2006, S. 210-233; Rychlo, Peter: Kafka-Rezeption in der deutschsprachigen Lyrik der Bukowina. In: Der literarische Zaunkönig. Nr. 3/2010. 2010, S. 10-20. Unter: http://www.erika-mitterer. org/dokumente/ZK2010-3/rychlo_kafka_3-2010.pdf [abgerufen am 09.10.2019]; Elm, Theo: Franz Kafka. In: May, Markus/ Peter Goßens/ Jürgen Lehmann (Hgg.): Celan-Handbuch. Leben-Werk-Wirkung. Stuttgart/Weimar: Metzler ${ }^{2} 2012$, S. 320-321.

${ }^{42}$ Poppe, Sandra: Die Verwandlung. In: Engel, Manfred/ Bernd Auerochs (Hgg): Kafka-Handbuch. Leben-Werk-Wirkung. Stuttgart / Weimar: J. B. Metzler 2010, S. 164-174, hier S. 171.

${ }^{43}$ Vgl. Poppe 2010, S. 172. 
des Sohnes Eric, nicht in die gemeinsame Wohnung zurückkehren. Er bezog folglich allein ein Einzimmer-Appartement in der Pariser Rue Tournefort und begab sich damit in eine private „Eis / heimat“, wie es im Gedicht Muschelhaufen heißt. ${ }^{44}$ Von der eigenen Familie quasi verstoßen schlüpfte Celan zwar nicht in den Panzer des Ungeziefers, wohl aber in die blaugefrorene Clownsmaske. Dabei verwendet er, wie Kafka, die Sprachfigur der Inversion, die bereits in Celans frühen Aphorismen erscheint und der er bis zu seinen späten Dichtungen treu bleiben sollte: ${ }^{45}$ „Winkelmund“ statt Mundwinkel, „Fragelocke Schwarz" statt schwarze Locke wie ein Fragezeichen. Und auch die Fortsetzung des Bildparadigmas der Kälte durch die ,großgestrählte, / großgespürte Rauhnacht", also eine der zwölf Nächte zwischen Weihnachten und Dreikönigsnacht (5/6. Januar $)^{46}$, mutet kafkaesk an. Das im Wort „großgestrählte“ angedeutete „Haarige“, das ebenfalls zur Etymologie von „Rauhnacht“" gehört ${ }^{47}$, scheint eine quasi surreale Vorstellung von den Brauen zu skizzieren, die wie „Riesenfühlerkämme“ die kalte und heilig-mystische Nacht durchkämmen. Der Gestus kann einen Zustand andauernder („Immerimmer") Suche wie auch der existenziellen Verlorenheit (,nicht hin, nicht her") in dieser groß gefeierten Nacht andeuten. Beides erinnert an Kafkas Kübelreiter in der gleichnamigen Erzählung oder an den Landarzt, den Celan wiederholt gelesen hat. Noch schmerzlichere Assoziationen

${ }^{44}$ Vgl. auch Wiedemann 2004, S. 176-177, 188.

${ }^{45}$ Vgl. Sparr 1988, S. 152-154.

${ }^{46}$ Die zwölf Verszeilen des Gedichts könnten dabei symbolisch auch für die zwölf Rauhnächte stehen. Vgl. Hünnecke, Evelyn: Aspekte der Sprechinstanz in dialogischer Lyrik. Paul Celans Gedicht Allmählich clowngesichtig. In: Cahiers d'études Germaniques. Qui parle dans le text? Études réunies par Maurice Godé et Marcel Vuillaume 38 (2000/1), S. 37-52, S. 42.

${ }^{47}$ Vgl. Fuß1 2008, S. 69: „Die Rauhnacht lässt sich etymologisch auf das althochdeutsche ,ruh ', was ,rau, grob, haarig, ungezähmt ${ }^{e}$ bedeutet, zurückführen.“ 
könnte eine alternative Bezeichnung der Rauhnächte als „Rauchnächte" beim Juden Celan hervorgerufen haben.

Mehrdeutig klingt auch die zuletzt genannte Bewegung im Vers: „schon fortgeschwungen aus der Flocke Welt“. Der semantische Spannungsbogen von „schwingen“ reicht von ,aufsteigen“, „,sich erheben“, „fliegen“ zu ,pendeln“, „,vibrieren“

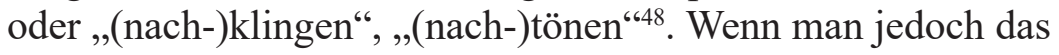
texttragende Bild der Clownsmaske oder des Clowngesichts bedenkt, so könnte die letzte Bewegung auf eine der gefährlichsten der Zirkuswelt verweisen: das vermeintlich leichte Schwingen eines Artisten am Trapez durch die Kuppel. Im Unterschied jedoch zum kunstvoll-akrobatischen Pendeln des Artisten in lebensgefährlicher Höhe legt das Fortschwingen Celans eine radikale existenzielle Bewegung nahe, die möglicherweise auf die trügerische Leichtigkeit des Lebens in der „Flocke Welt" aber auch jene der totalen Vereinsamung oder des Sterbens verweist.

Zusammenfassend kann man Fuß1 teilweise zustimmen: „Allmählich clowngesichtig ist die Topographie einer Gesichtslandschaft mit Mund, Schädel und Augenbrauen. “49 Genauer ist es die Topographie einer Maske oder eines maskenhaften Gesichts. Nicht zustimmen kann man dem Befund, dass diese Mimik nichts spiegelt und Gefühle lediglich vorgaukelt. ${ }^{50}$ Denn wie im Falle Kafkas ist hier die Maske oder das maskenhafte Gesicht eine ambivalente Larve, die zeigt, indem sie verbirgt. Sie ist der bildliche Ausdruck einer seelischen Befindlichkeit, der bitteren Enttäuschung über die Ohnmacht der Wahrheit, der eisigen Kälte der Vereinsamung und last but not least der Groteske des Daseins in einer moralisch oberflächlichen Welt. Durch die Maske des tragischen, weil illusionslosen Clowns

\footnotetext{
${ }^{48}$ Vgl. Duden: schwingen. https://www.duden.de/rechtschreibung/ schwingen [20.10.2019]

${ }^{49}$ Fuß1 2008, S. 71.

${ }^{50}$ Ebenda.
} 
artikuliert Celan nicht nur ein individuelles Bewusstsein sondern auch eine moderne, facettenreiche, spielerische, oft tragische Identität, die sich schon seit dem Symbolismus künstlerisch in der Maske des Narren zeigt und zugleich verbirgt.

\section{Literaturverzeichnis}

\section{Primärliteratur}

Bachmann, Ingeborg/ Paul, Celan: Herzzeit. Der Briefwechsel, hrsg. u. kommentiert von Bertrand Badiou, Hans Höller, Andrea Stoll und Barbara Wiedemann, Frankfurt am Main: Suhrkamp ${ }^{5} 2016$.

Celan, Paul: Lichtzwang. Vorstufen-Textgenese-Endfassung. Bearbeitet von Heino Schmull unter Mitarbeit von Markus Heilmann und Christiane Wittkop, Frankfurt am Main: Suhrkamp 2001.

Celan, Paul: Die Gedichte. Kommentierte Gesamtausgabe in einem Band. Hrsg. v. Barbara Wiedemann, Frankfurt am Main: Suhrkamp 2003.

Celan, Paul/ Dischner, Gisela: Wie aus weiter Ferne zu Dir.

Briefwechsel. Hrsg. v. Barbara Wiedemann, Frankfurt am

Main: Suhrkamp 2012.

Sekundärliteratur

Amthor, Wiebke: Schneepart. In: May, Markus/ Peter Goßens/ Jürgen Lehmann (Hgg.): Celan-Handbuch. Leben - Werk - Wirkung. Stuttgart / Weimar: J. B. Metzler ${ }^{2}$ 2012, S. 115-123.

Barloewen, Constantin: Clown. In: Hügel, Hans-Otto (Hg.): Handbuch Populäre Kultur. Begriffe, Theorien und Diskussionen. Stuttgart/Weimar: J. B. Metzler 2003, S. 134-138. Barloewen, Constantin von: Der Clown als konstruktiver Anarch. Reflexionen über die Dialektik des Clowns. Constantin Barloewen im Gespräch mit Rafiu Raji und Richard Weihe. In: Weihe, Richard (Hg.): Über den Clown. 
Künstlerische und theoretische Perspektiven. Bielefeld: transcript Verlag 2016, S. 127-136.

Behringer, Wolfgang: Kulturgeschichte des Klimas: Von der Eiszeit bis zur globalen Erwärmung. München: C.H. Beck 52010.

Bogumil-Notz, Sieghild: Lichtzwang. In: May, Markus/ Peter Goßens/ Jürgen Lehmann (Hgg.): Celan-Handbuch. Leben - Werk - Wirkung. Stuttgart / Weimar: J. B. Metzler 22012, S. 105-115.

Cheie, Laura: Harte Lyrik. Zur Psychologie und Rhetorik lakonischer Dichtung in Texten von Günter Eich, Erich Fried und Reiner Kunze. Innsbruck: Studienverlag 2010.

Daphinoff, Dimiter: Shakespeares Narren. In: Huber, H. (Hg.): Der Narr: Beiträge zu einem interdisziplinären Gespräch. Freiburg, Schweiz: Univ.-Verl. 1991 (= Studia ethnographica Friburgensia. Bd. XVII), S. 57-69.

Elm, Theo: Franz Kafka. In: May, Markus/ Peter Goßens/ Jürgen Lehmann (Hgg.): Celan-Handbuch. Leben-WerkWirkung. Stuttgart/Weimar: Metzler ${ }^{2} 2012$, S. 320-321.

Felstiner, John: Paul Celan. Eine Biographie. Deutsch von Holger Fliessbach. München: C.H.Beck 1997.

Fried, Annette / Joachim, Keller: Faszination Clown. Düsseldorf: Patmos 1996.

Fuß1, Irene: „Geschenke an Aufmerksame“. Hebräische Intertextualität und mystische Weltauffassung in der Lyrik Paul Celans. Tübingen: Niemeyer 2008.

Günzel, Elke: Das wandernde Zitat. Paul Celan im jüdischen Kontext. Würzburg: Königshausen \& Neumann 1995.

Hartung, Harald: Masken und Stimmen. Figuren der modernen Lyrik. München/Wien: Hanser 1996.

Horn, Peter: Die Garne der Fischer der Irrsee. Zur Lyrik von Paul Celan. Oberhausen: Athena 2011.

Hünnecke, Evelyn: Aspekte der Sprechinstanz in dialogischer Lyrik. Paul Celans Gedicht „Allmählich clowngesichtig“. In: Cahiers d'études Germaniques. Qui parle dans le text? 
Études réunies par Maurice Godé et Marcel Vuillaume 38 (2000/1), S. 37-52.

Liska, Vivian: Ein Meridian wider die Zeit. Von Celan zu Kafka. In: Engel, Manfred/ Dieter Lamping (Hgg.): Franz Kafka und die Weltliteratur. Göttingen: Vandenhoeck \& Ruprecht 2006, S. 210-233.

Poppe, Sandra: Die Verwandlung. In: Engel, Manfred / Bernd Auerochs (Hgg): Kafka-Handbuch. Leben - Werk - Wirkung. Stuttgart / Weimar: J. B. Metzler 2010, S. 164-174. Pöggeler, Otto: Spur des Worts. Zur Lyrik Paul Celans. München: Karl Alber 1986.

Pöggeler, Otto: Der Stein hinterm Aug. Studien zu Celans Gedichten. München: Fink 2000.

Solomon, Petre: Paul Celan. Dimensiunea românească.

Bukarest: Kriterion 1987.

Sparr, Thomas: Celan und Kafka. In: Celan-Jahrbuch 2 (1988),

S. 140-154.

Starobinski, Jean: Porträt des Künstlers als Gaukler. Drei Essays. Aus dem Französischen von Markus Jacob. Frankfurt am Main: S. Fischer 1985.

Velten, Hans Rudolf: Spaßmacher. In: Wirth, Uwe (Hg.): Komik. Ein interdisziplinäres Handbuch. Stuttgart: Metzler 2017, S. 42 - 46.

Vietta, Silvio: Die literarische Moderne. Eine problemgeschichtliche Darstellung der deutschsprachigen Literatur von Hölderlin bis Thomas Bernhard. Stuttgart: J. B. Metzler 1992.

Vietta, Silvio: Die Modernekritik der ästhetischen Moderne. In: Vietta, Silvio/ Dirk Kemper (Hgg.): Ästhetische Moderne in Europa. Grundzüge und Problemzusammenhänge seit der Romantik. München: Fink 1998, S. 531-549.

Weihe, Richard: Die Paradoxie der Maske. Geschichte einer Form. München: Fink 2004.

Weihe, Richard: Das (Un-)Behagen am Clown. Einleitung. In: Weihe, Richard (Hg.): Über den Clown. Künstlerische und 
theoretische Perspektiven. Bielefeld: transcript Verlag 2016a, S. 7-21.

Weihe, Richard: Die Paradoxie des Clowns - sieben Spielformen. In: Weihe, Richard (Hg.): Über den Clown. Künstlerische und theoretische Perspektiven. Bielefeld: transcript Verlag 2016b, S. 265-274.

Werner, Uta: Textgräber. Paul Celans geologische Lyrik. München: Fink 1998.

Wiedemann, Barbara: Paul Celan - Die Goll-Affäre: Dokumente zu einer „Infamie“. Frankfurt am Main: Suhrkamp 2000.

Wiedemann, Barbara: „LESEN SIE! IMMERZU NUR LESEN": Celan-Lektüre und Celans Lektüren. In: „Poetica“. Bd.36, Nr. 1/2 (2004), S. 169-191.

Internetquellen

Rychlo, Peter: Kafka-Rezeption in der deutschsprachigen Lyrik der Bukowina. In: Der literarische Zaunkönig. Nr. 3/2010. 2010, S. 10-20. Unter: http://www.erika-mitterer. org/dokumente/ZK2010-3/rychlo_kafka_3-2010.pdf [Abgerufen am 09.10.2019]

Blumen aus der Eiszeit. In: Die Zeit, Nr. 47/1967: https:// www.zeit.de/1967/47/blumen-aus-der-eiszeit/komplettansicht [03.10.2019]

Eine Eiszeit pro kosmisches Jahr. In: Die Zeit, Nr. 51/1967, https://www.zeit.de/1967/51/eine-eiszeit-pro-kosmischesjahr [03.10.2019]

Duden: Clown. https://www.duden.de/rechtschreibung/Clown [20.10.2019]

Duden: schwingen. https://www.duden.de/rechtschreibung/ schwingen [20.10.2019] 